\title{
The Universality of Experiential Consciousness
}

\author{
Robert K. Logan $\mathbb{D}$ \\ Department of Physics, University of Toronto, Toronto, ON M5S 1A, Canada; logan@physics.utoronto.ca
}

Received: 16 November 2018; Accepted: 11 January 2019; Published: 17 January 2019

\begin{abstract}
It is argued that of Block's (On a confusion about a function of consciousness, 1995; The Nature of Consciousness: Philosophical Debates, 1997) two types of consciousness, namely phenomenal consciousness (p-consciousness) and access consciousness (a-consciousness), that $\mathrm{p}$-consciousness applies to all living things but that a-consciousness is uniquely human. This differs from Block's assertion that a-consciousness also applies to some non-human organisms. It is suggested that p-consciousness, awareness, experience and perception are basically equivalent and that human consciousness has in addition to percept-based p-consciousness, concept-based a-consciousness, a verbal and conceptual form of consciousness that can be utilized to coordinate, organize and plan activities for rational decision-making. This argument is based on Logan's (The Extended Mind: The Emergence of Language, The Human Mind and Culture, 1997) assertion that humans are uniquely capable of reasoning and rationality because they are uniquely capable of verbal language and hence the ability to conceptualize.
\end{abstract}

Keywords: consciousness; experience; phenomenal consciousness; access consciousness; percept; concept; language

Consciousness is a mongrel concept-Ned Block [1]

Sentience is consciousness_Eugene T. Gendlin [2]

Anything that we are aware of at a given moment forms part of our consciousness, making conscious experience at once the most familiar and most mysterious aspect of our lives-Susan Schneider and Max Velmans [3].

\section{Introduction}

As Schneider and Velmans [3] have stated, consciousness is something we are constantly aware of but find difficult to understand or explain. The perceived mystery of consciousness has been so great that many in the past and some today have denied its existence and considered the study of it a fool's errand. In more recent times this skepticism has abated and it is considered to be a legitimate area of study by many researchers in philosophy, psychology, cognitive science and neuroscience.

The mystery associated with consciousness is finding a way to explain what makes it possible as well as providing answers to the following questions: How is it connected to language and thought? Are non-human animals conscious and to what extent? What is the relationship of consciousness to awareness, perception, experience, conceptualization and thought?

\section{Block's Phenomenal Consciousness and Access Consciousness}

Ned Block [1] has suggested that there are two types of consciousness, namely, phenomenal consciousness ( $\mathrm{p}$-consciousness) and access consciousness (a-consciousness).

- Phenomenal consciousness is experience; the phenomenally conscious aspect of a state is what it is like to be in that state. The mark of access-consciousness, by contrast, is availability for use in reasoning and rationally guiding speech and action [1] (p. 227). 
- P-conscious states are experiential, that is, a state is $\mathrm{p}$-conscious if it has experiential properties. The totality of the experiential properties of a state are 'what it is like' to have it. Moving from synonyms to examples, we have p-conscious states when we see, hear, smell, taste, and have pains [4].

- I agree with Block that all living organisms possess some level of p-consciousness in that they are aware of their environment and experience their surrounding through their channels of perception. Where I disagree with Block is that a-consciousness can occur with non-human organisms. In particular I take issue with the following assertion that Block makes:

A state is access- conscious (A-conscious) if, in virtue of one's having the state, a representation of its content is (1) inferentially promiscuous (Stich 1978), that is, poised for use as a premise in reasoning, (2) poised for rational control of action, and (3) poised for rational control of speech. (I will speak of both states and their contents as A-conscious.) These three conditions are together sufficient, but not all necessary. I regard (3) as not necessary (and not independent of the others), because I want to allow that non- linguistic animals, for example chimps, have A-conscious states. I see A-consciousness as a cluster concept, in which (3) - roughly, reportability - is the element of the cluster with the smallest weight, though (3) is often the best practical guide to A-consciousness [1], [bolding is mine].

I believe there is value in Block's formulation of a- and p- consciousness but I also believe that conceptualization is intimately connected to and dependent on verbal symbolic language, a position I share with others including Brandom [5,6], Davidson [7] and Dummett [8]. The issue of whether or not conceptualization requires language and whether non-human animals are capable of conceptualization is not resolved and perhaps cannot be resolved scientifically because the falsification of the proposition is not possible since we cannot get into the minds of non-human animals as we can with fellow humans through our theory of mind. A theory of mind depends on the assumption that other humans think as I do and that they, like me, have thoughts, reasonings, interests, desires, and intentions similar to mine. My theory of mind is based on my social interactions with other humans and is more precise with those with whom I share a common language and culture than with those from a different language and cultural group. There are limits to my theory of mind as I am not always able to predict the reactions to what I say and do with others even with members of my own family. It is also certainly the case that I and other fellow humans have no theory of mind of non-human animals nor can we. It is, therefore, impossible to know how they think or even if their behavior is guided by thought.

Certainly a-consciousness cannot be available "for use in reasoning and rationally guiding speech" for non-humans as only humans are capable of speech. As for its availability "for use in reasoning and rationally guiding ... action" for non-human beings, I believe that this is not possible because reasoning and rationality depends on the ability to conceptualize. I have argued elsewhere that conceptualization, the basis for reasoning and rationality depends on the possession of verbal language [4], a unique feature of humans. I will also argue that Block's p-consciousness is percept-based as it is experiential and that a-consciousness is concept-based as it is associated with reasoning and rationality.

\section{The Many Levels of Experiential or Phenomenal Consciousness (P-Consciousness)}

Percept-based or p-consciousness is based on the awareness or perception of one's sensations and hence all forms of life have, to a certain extent, some form of consciousness. Percept-based or p- consciousness is nothing more than the awareness of one's perceptions. The simplest bacteria are able to distinguish food from toxins and move towards the former and away from the latter. This is a very primitive form of $\mathrm{p}$-consciousness. One may say that bacteria are conscious or aware of food and toxins and act accordingly. This form of consciousness is chemical in nature.

There are many levels of percept-based p-consciousness including chemical, tactile, pain, thermoperception, kinesthetic, magnetic, auditory, olfactory, gustatory, equilibriception and visual forms of consciousness and each form of p-consciousness corresponds to its respective sensory channel or capability. P-consciousness is about an organism perceiving, sensing or experiencing environmental signals from its umwelt [9] as well as internal signals. 
Chemical forms of consciousness involve the pheromones of prokaryotes, slime molds, plants, and even with higher forms of life such as social insects like ants and some vertebrates. Pheromones can signal alarms, food possibilities, mating opportunities, etc. Another form of chemical signaling is quorum detection in bacteria, single-cell eukaryotes, fungi and social insects in which the density of conspecifics can affect gene expression for single-cell organisms. These organisms are conscious of the con-specifics in their colony.

Other levels of consciousness include the following:

- Tactile consciousness is where organisms with a primitive sensory apparatus are aware of touch or pain or heat.

- Auditory consciousness involves sound signals such as tones, growls, whines, barks, hoots, bird songs, mating calls, and for humans, verbal language as well as non-verbal prosody.

- Olfactory consciousness involves the smell of flowers for insects and birds and the smell of prey for raptors or the smell of a potential mate for a variety of animals.

- Gustatory consciousness involves the tasting mechanism of the tongue and epiglottis of many different animal species.

- Magnetoception consciousness involves the detection of the earth's magnetic field for navigation purposes by organisms including bacteria, insects, lobsters, stingrays, turtles, and birds.

- Visual consciousness involves the detection of visual signals.

- Thermoperception consciousness includes sensitivity to temperature, proprioception for a kinesthetic sense, nociception for pain and equilibrioception for balance experienced by a wide variety of animals including us humans.

- A level of perception and hence the consciousness of plants exists including their awareness of light, moisture, temperature, gravity, touch (in the case of carnivorous plants and mimosa pudica), and chemical signals from other plants.

- Given that percept-based or p-consciousness is characteristic of the full range of living organisms, one can conclude that the reaction to this form of consciousness is instinctive (private communication Alice Braga Gastaldo) as opposed to concept-based a-consciousness, which can be acted upon with rational forethought.

\section{Percept-Based and Concept-Based Thinking}

Because consciousness is a sentient phenomenon before addressing the nature of percept and concept-based consciousness, i.e. p- and a- consciousness, I will first review my study of the emergence of language in The Extended Mind: The Emergence of Language, the Human Mind and Culture [4], where I make a distinction between perceptual and conceptual thinking. Concept-based thinking is associated with verbal language and is unique to human beings. In my study, I suggested that words and concepts co-emerged with humans. It is proposed that the origins of speech and the human mind emerged simultaneously as the bifurcation from percept-based to concept-based mental activity. This transition was a response to the chaos associated with the information overload that resulted from the increased complexity in hominin life. This complexity arose in part with the hominin control of fire that led to hominins living in large groups instead of nuclear family units in order to share the many benefits of fire. Living in large groups required the coordination of many individuals and the necessity of planning. It is surmised that the hominin brain could no longer cope with the richness of life solely on the basis of its perceptual sensorium and as a result a new level of order emerged in the form of conceptualization and speech. Speech arose as a way to control information and was also used as a medium for communication. "Rather than regarding speech as vocalized thought one may just as well regard thought as silent speech [4] (p. 5)."

The mechanism that allowed the transition from percept-based to concept-based mental activity was the emergence of speech. The words of spoken language are the actual medium or mechanism by which concepts are expressed or represented (actually re-presented). Words are both metaphors and 
strange attractors uniting many perceptual experiences in terms of a single concept. For example, the word 'water' is a strange attractor for all of our percepts of water, namely the water we drink, cook with, wash with, falls as rain, and is found in rivers, ponds, lakes and oceans. Spoken language and abstract conceptual thinking emerged simultaneously at exactly the same point of time as a bifurcation from the concrete percept-based thinking of pre-lingual hominins to the abstract concept-based thinking of Homo sapiens. It is suggested that it might also have been the moment that Homo sapiens or full humans first emerged from their hominin ancestors.

Before humans acquired language, their brain was a percept processor. With language, the mind emerged as a conceptualization engine so that the mind is the product of the brain and verbal language (mind $=$ brain + language). Because the mind is capable of conceptualization it allowed for planning and complex social organization, all of which aided human survival.

For non-human organism sentience is percept based and is a universal property of all living organisms from the simplest bacteria to our hominin ancestors. Human beings, on the other hand, have both percept-based and concept-based sentience. As consciousness and sentience are essentially the same as Eugene T. Gendlin [2] has asserted, I suggest that all living organism are conscious in that they possess p-consciousness or percept-based consciousness. This is a rather bold statement but it is based on the work of Terrence Deacon [10] in his book Incomplete Nature. The argument for the universality of p-consciousness follows from Deacon's notion that all living organisms are teleodynamic systems or selves that operate in their own self-interest. In order to act in their own self-interest a living organism has to be conscious, i.e. aware of its surrounding and its internal state so that it can take the appropriate action to insure it is operating in its own self-interest. An agent cannot realize its purpose if it is not conscious of those things that bear on its wellbeing. P-consciousness or percept-based consciousness is nothing more than a living organism being aware of or experiencing something happening within their environment or within their body.

A-consciousness or concept-based consciousness is strictly restricted to human beings because we are the only organism capable of verbal language and hence conceptualization. A-consciousness is therefore being aware of our thoughts and knowing what we know and hence is basically listening to ourselves silently talking to ourselves. So, for me the really hard problem of consciousness is how we developed verbal language so necessary for a-consciousness. Unless we translate our percepts or the products of our p-consciousness into concepts or silent language they are not accessible "for use in reasoning and rationally guiding speech and action" and as such will not be part of our a-consciousness.

Despite attempts to explain the origin of language, we still do not have a totally satisfactory description of this phenomenon and hence not one of a-consciousness either. Our various explanations for the origin of language and hence a-consciousness are "just-so" stories or abductions, including my own [4]. This is why I claim that a-consciousness is a harder problem to understand than p-consciousness.

\section{Complexity and Emergence and the Transition from Percept-Based Thinking and Consciousness to Concept-Based Thinking and Consciousness}

I suggested above that it was the complexity of living together in clans instead of nuclear family groupings that gave rise to the emergence of verbal language and concept-based thinking and consciousness. I chose the words complexity and emergence purposely because I believe that this transition can be explained in terms of complexity theory, which grew out of general systems theory. Systems theory is the notion that a system must be analyzed in terms of the system as a whole and not as the sum of the components making up the system. Complexity theory concerns itself with the various interactions and feedback loops of the components of the system with each other and with the system as a whole. In a complex system the behavior of the system and its components are the simultaneous result of the top-down causes of the system on its components, the bottom-up causes of the components on the supervenient system and the lateral causes of the components on each other. 
This results in the supervenient system having properties that none of its components have. In simple terms, the system is more than the sum of its parts.

I would suggest that living in a complex community where cooperation was key to survival through the maintenance of the camp fire that there emerged out of the complexity of human interactions the ability to communicate and formulate thoughts in the abstract medium of verbal language and conceptual thinking. Just as spoken language allows a community to coordinate, organize and plan its activities, silent speech or thought or a-consciousness allows individuals to coordinate, organize and plan their activities. Given that speech is vocalized thought and thought is silent speech, speech and thought are basically the same phenomena. Therefore, a-consciousness or thought is nothing more than listening to one's silent speech. A-consciousness is in a certain sense being aware of one's awareness and that requires thought in the form of silent speech to achieve.

Since non-humans are not capable of verbal language, I therefore conclude that non-humans are unaware of their thought processes and therefore their level of consciousness cannot match that of humans. This is a non-scientific proposition since we have no way of knowing how non-humans think or what they think about. Some would claim they do not think but only operate by instinct but I believe there is enough evidence that indicates that some non-human animals do indeed think given their ability to solve certain problems. I do not believe, however, that they possess a-consciousness. This is purely speculative as our theory of mind only extends to other humans who we assume act and think the way we do. I cannot assume a non-human thinks the way I do.

\section{Discussion and Conclusion}

I find Block's distinction between p-consciousness and a-consciousness very useful. My qualification of his work is that while p-consciousness is universal for all forms of living organisms, a-consciousness is not. I have argued that only humans are capable of a-consciousness because their ability to use language allows them to conceptualize and hence 'reason and rationally guide action,' one of Block's criteria for a-consciousness. I am ready to concede, however, that perhaps some other forms of life might be capable of conceptualization such as non-human primates based on the observations and experiments with non-human primates solving problems and communicating using words. The cases of Washoe who learned to use 350 ASL signs [11] and Kanzi who learned language through the use of a keyboard lexigram [12] are prime example. On the other hand, I am quite certain that bacteria, single cell eukaryotes, fungi and plants are not capable of a-consciousness. The question becomes where does on draw the line between those organisms capable of a-consciousness and those that are not. This is a question for those that study non-human animal behavior.

Funding: This research did not receive any specific grant from funding agencies in the public, commercial, or not-for-profit sectors.

Conflicts of Interest: The author declares no conflict of interest.

\section{References}

1. Block, N. On a confusion about a function of consciousness. Behav. Brain Sci. 1995, 18, 227-287. [CrossRef]

2. Gendlin, E.T. Implicit precision. In Knowing without Thinking: Mind, Action, Cognition and The Phenomenon of the Background; Radman, Z., Ed.; de Gruyter: Amsterdam, the Netherlands, 1995; p. 148.

3. Schneider, S.; Velmans, M. Introduction. In The Blackwell Companion to Consciousness; Velmans, M., Schneider, S., Eds.; Wiley: New York, NY, USA, 2008.

4. Logan, R.K. The Extended Mind: The Emergence of Language, The Human Mind and Culture; University of Toronto Press: Toronto, Canada, 2007.

5. Brandom, R. Making It Explicit: Reasoning, Representing, and Discursive Commitment; Harvard University Press: Cambridge, MA, USA, 1994.

6. Brandom, R. Articulating Reasons: An Introduction to Inferentialism; Harvard University Press: Cambridge, MA, USA, 2000. 
7. Davidson, D. Thought and Talk. Inquiries into Truth and Interpretation; Oxford University Press: Oxford, UK, 1975.

8. Dummett, M. Seas of Language; Oxford University Press: Oxford, UK, 1993.

9. Von Uexküll, J. Theoretical Biology; Harcourt, Brace \& Co.: New York, NY, USA, 1926.

10. Deacon, T. Incomplete Nature: How Mind Emerged from Matter; Norton: New York, NY, USA, 2012.

11. Gardner, R.A.; Gardner, B.T. The Structure of Learning from Sign Stimuli to Sign Language; Lawrence Erlbaum Associates: Hillsdale, NJ, USA, 1998.

12. Savage-Rumbaugh, S.; Lewin, R. Kanzi: The Ape at the Brink of the Human Mind; Wiley: New York, NY, USA, 1994.

(C) 2019 by the author. Licensee MDPI, Basel, Switzerland. This article is an open access article distributed under the terms and conditions of the Creative Commons Attribution (CC BY) license (http:// creativecommons.org/licenses/by/4.0/). 